\title{
Coupling Multi-Scale Mechanical Testing Techniques with FIB and TEM Characterization to Reveal Mechanisms of Embrittlement of High Dose Inconel X-750
}

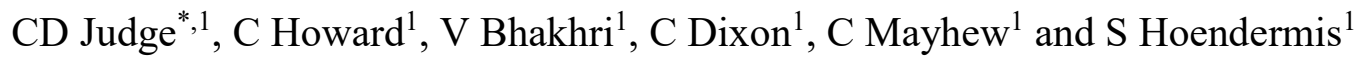 \\ 1. Canadian Nuclear Laboratories, Research and Development, Chalk River, Ontario, Canada. \\ * Corresponding author: colin.judge@cnl.ca
}

In this study, advanced microscopy techniques are used to investigate bulk mechanical testing and small scale testing of heavily irradiated Inconel X-750 to elucidate the mechanisms of embrittlement and fracture. When subjected to a high thermal flux, the naturally occurring Ni-58 in X-750 undergoes transmutation reactions, $(n, \alpha),(n, p)$ and $(n, \gamma)$, which results in an enhancement of the total atomic displacement damage (dpa), and the production of high concentrations of Helium and Hydrogen [1]. Material in this investigation has been irradiated up to $84 \mathrm{dpa}$ and $26000 \mathrm{appm}$ Helium. Bulk component testing has shown that the material has lost strength and ductility following irradiation, and that the fracture surfaces are brittle, mixed mode, which are predominantly intergranular, with evidence of transgranular facets (Figure 1 a) [2, 3]. In-situ, small scale mechanical testing has been performed in attempt to assess the grain boundary strength $[2,4]$; however, the grain boundaries were found to be inherently stronger than the bulk material $[2,4]$, and the easiest fracture path occurs exclusively along flow localization pathways (i.e., shear bands) consistent with the transgranular facets observed in the bulk fracture surfaces in Figure 1 a.

To ascertain the specific mechanisms of embrittlement and fracture, a focused ion beam (FIB) was used to extract transmission electron microscopy (TEM) specimens directly from both intergranular, and transgranular fracture surfaces (Figure 1 b \& c). The subsequent TEM examination has revealed evidence to support two fracture mechanisms. The synergies of the two have been shown to result in the accumulating degradation of the material.

The intergranular fracture is a direct result of accumulated helium bubbles aligned along grain boundaries, and carbide-matrix interfaces (Figure $1 \mathrm{~d}$ ). In addition to observing the interlinking of helium bubbles and resulting crack propagation at the crack tip, independent bubble coalescence and fracture ahead of the crack tip was observed. Along the entire fracture surface and secondary cracks, shear bands in the grain interior were found intersecting the grain boundary. It is important to note that no such shear bands are observed in as irradiated, non-deformed material. This deformation seems to be a predecessor to cracking. It is hypothesized that the intersection of these slip bands with the compromised grain boundary decorated with $\mathrm{M}_{23} \mathrm{C}_{6}$ carbides and grain boundary helium bubbles, resulted in a stress concentration, and assisted in the crack propagation.

The transgranular fracture seems to follow shear bands within the grain interior. Figure 1 e shows a high resolution TEM micrograph imaged close to the (111) zone axis. The image provides direct evidence of the mechanism of bubble elongation following subsequent deformation within the grain interior. Although not observed from these TEM specimens, it can be hypothesized that elongated helium bubbles may eventually shear to the point of coalescing to form large bubble ligaments, and ultimately result in the transgranular facets observed in the bulk fracture surfaces as has already been seen in the TEM examinations of small-scale mechanical test specimens [2]. What remains unknown, is 
the interaction between these flow localizations and the embrittled boundaries decorated with helium bubbles (i.e., the synergies of the two mechanisms). To answer this question, a small scale test specimen with a vertical boundary along the gage length has been tested (Figure 2). This style of test forces the shear bands to hit the boundary such that the interaction can be examined with subsequent microscopy. Transmission electron microscopy (TEM) of the deformed micro-tensile specimens has confirmed both the fracture along discrete shear bands within the bulk material, and the nucleation of cracking at the shear band and grain boundary carbides. As expected, more extensive strain localization was observed in the small scale test specimens (Figure 2) compared to the bulk material (Figure 1 e) with more significant bubble elongation; however, the observations remain consistent.

\section{References:}

[1] CD Judge et al., in „Embrittlement of Nickel Alloys in a CANDU* Reactor Environment“ Ed. T. Yamamoto (ASTM International, Anaheim) p. 161.

[2] C Howard et al., Journal of Nuclear Materials 517 (2019), p. 17.

[3] CD Judge et al., Journal of Nuclear Materials 457 (2015), p. 165.

[4] C Howard, CD Judge and P Hosemann, Materials Science and Engineering: A 748 (2019), p. 396.

(a)

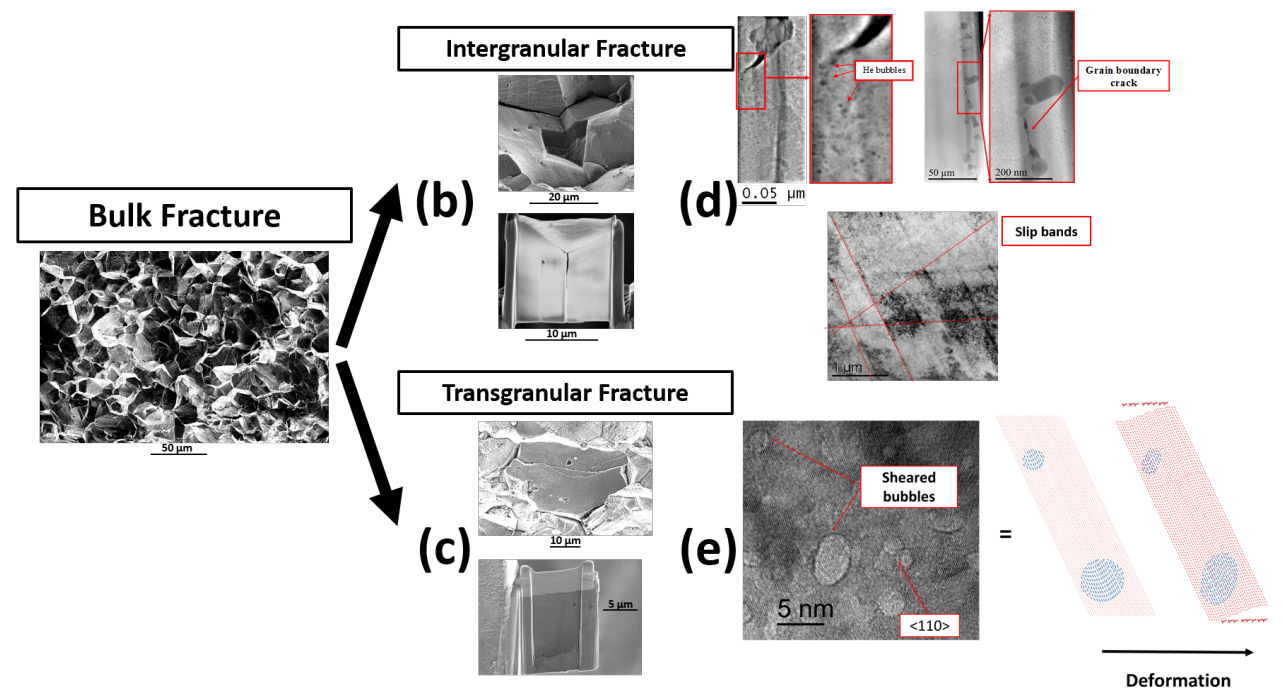

Figure 1. Examination of (a) bulk mechanical testing fracture surface, (b) FIB lift-out of an intergranular fracture surface, (c) FIB lift-out of a transgranular fracture surface, (d) TEM evidence of grain boundary helium bubble embrittlement, and shear band formation following subsequent deformation, (e) evidence of dislocation shearing of helium bubbles.
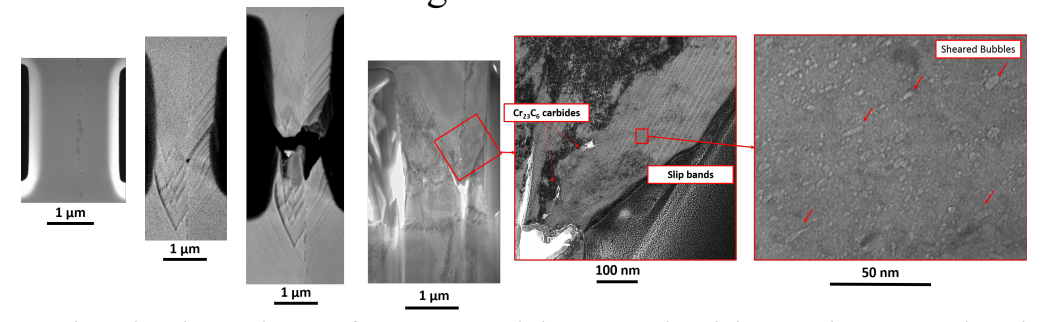

Figure 2. Small scale mechanical testing of $X-750$ with a vertical boundary, and subsequent TEM examination after deformation and fracture. 